\title{
Greffe osseuse sinusienne à visée implantaire
}

\section{Sinus bone grafts for implantation purposes}

\section{MOTS-CLEFS : \\ - Implant, sinus, greffe osseuse, élévation sinusienne. \\ KEYWORDS: \\ - Implant, sinus, bone graft, sinus lift.}

AOS 2013;264:20-26 DOI: $10.1051 /$ aos/2013405 C) EDP Sciences 2013

\section{Résumé}

Actuellement, la demande de prothèse implanto-portée est en constante augmentation, ce qui nécessite un volume osseux suffisant et donc le recours à des apports osseux dans certains cas.

Les apports osseux peuvent se faire par de l'os autogène ou des biomatériaux. Il n'y a pas encore de consensus quant au choix des greffes à utiliser. Aussi, après avoir exposé les indications et les différentes techniques utilisées, nous vous présenterons des cas traités avec leurs résultats.

Cet article n'a aucune prétention ò être exhaustif. En effet, le chirurgien, qu'il soit maxillo-facial ou chirurgien-dentiste, doit connaître les indications, les contreindications, les techniques et la prise en charge des complications. Comme dans tous les actes chirurgicaux, l'expérience du chirurgien est un atout évident.

\section{Abstract}

At present, there is ever-growing demand for implant-supported prostheses, which require a sufficient volume of bone and therefore, in some cases, require additional bone.

Additional bone can be provided in the form of autogenic bone or biomaterials. There is not yet a consensus about which type of graft to use. Therefore, after having presented the indications and the various different techniques used, we will present some cases treated and their outcomes.

This article does not set out to be exhaustive. The surgeon, whether (s)he is a maxillo-facial surgeon or a dental surgeon, must be aware of the indications, contraindications, techniques and how to deal with complications. As in all surgical procedures, the surgeon's experience is an obvious asset.

\section{Dominique Deffrennes, Chirurgien Maxillo-facial et Plastique, 2 rue St Pétersbourg, 75008 Paris}

Georges Khoury, Elias Khoury, Docteurs en Chirurgie Dentaire, 83 boulevard Exelmans, 75016 Paris

Thierry Guérin, Docteur en chirurgie dentaire, ex assistant Paris V, 25 rue des Petits Hôtels, 75010 Paris

\section{RAPPEL DE L'ANATOMIE ET DE LA PHYSIOLOGIE}

Une connaissance de l'anatomie et de la physiologie est indispensable comme dans toute chirurgie.

Le sinus maxillaire présente une paroi nasale, orbitaire, jugale, infratemporale et inférieure avec son processus alvéolaire. Le volume du sinus est variable et parfois s'insinue dans les racines dentaires [1]. Les pertes dentaires peuvent s'accompagner d'importantes pertes osseuses ou en tous les cas d'insuffisance de volume osseux contre-indiquant la pose d'implants vissés. Les irrégularités du plancher sinusien, les cloisons verticales intrasinusiennes, la finesse membranaire sinusienne et les pertes osseuses complètes rendent difficile le décollement membranaire et donc la mise en place de la greffe [2]. La nécessité d'un bilan radiologique 
avant l'intervention se comprend aisément.

La membrane sinusienne recouvre les parois du sinus et est en continuité avec la muqueuse nasale. Cette membrane n'a pas les caractéristiques du périoste et ne semble pas présenter d'ostéoblaste. Cependant, certaines études montrent la formation d'os lors de levées de membrane en l'absence de tout apport.

Les parois du sinus sont vascularisées par les branches de l’artère maxillaire. Les incisions de décharge au niveau de la grosse tubérosité sont plus hémorragiques que celles situées en mésial de la première prémolaire du fait des branches descendantes de l'artère infra-orbitaire. En revanche, l'artère alvéolo-antrale horizontale intra-osseuse ou à l'interface muqueuse-os peut, en cas de lésion chirurgicale, gêner considérablement le chirurgien lors du décollement de la muqueuse sinusienne. Cette artère est d'autant plus petite, voire insignifiante, que le sujet est âgé et/ou édenté depuis longtemps. Cette branche artérielle doit être repérée sur le scanner avant l'acte chirurgical, généralement située entre le tiers inférieur et moyen de la paroi sinusienne antérieure donc souvent au-dessus de la fenêtre osseuse nécessaire pour la mise en place de la greffe et la pose d'implants. Le décollement de la membrane sur la face nasale peut provoquer un saignement du fait des branches descendantes de l'artère sphénopalatine. La paroi inférieure est en revanche peu vascularisée [3]. Le méat moyen permet au sinus d’être drainé à l'aide de la membrane ciliée qui tapisse le sinus. Aussi toutes les anomalies de cet ostium peuvent être responsables de sinusite contre-indiquant la mise en place d'une greffe osseuse, d'où le recours à un chirurgien ORL.

La connaissance de l'innervation, branches du nerf maxillaire (V2), oriente les points d'anesthésie locale. Généralement, l'infiltration intéresse la région parapicale de la canine à la tubérosité et la région palatine postérieure [4]. L'infiltration évitera le contact avec le périoste pour ne pas léser les branches artérielles alvéolaires.

\section{INDICATIONS ET CONTRE- INDICATIONS DE LA GREFFE DANS LE SINUS MAXILLAIRE}

Les indications de la greffe dépendent du volume osseux résiduel après les extractions dentaires. La résorption osseuse se fait dans le sens vertical et transversal de la crête osseuse [5]. La résorption est plus importante au niveau vestibulaire. De nombreuses classifications ont été décrites [6]. Ces classifications tiennent compte de la hauteur et de la largeur de la crête résiduelle ainsi que de la hauteur de la crête à la jonction émail-cément de la dent adjacente à lédentement. Cette dernière hauteur définit l'espace inter-arcade et donc le rapport couronne-racine qui peut être défavorable si cet espace est trop élevé. En fonction de ces critères, l'association de comblement sinusien, de greffe d'apposition en " onlay » et de la régénération osseuse guidée peut être nécessaire.
De nombreuses études ont été publiées concernant les greffes sinusiennes qui ne permettent pas d'obtenir des certitudes scientifiques. En fait, il faudrait faire des études prospectives avec un suivi sur cinq ans pour pouvoir aboutir à des conclusions fiables. Les études restent trop empiriques, aussi nous vous indiquerons notre attitude sur les différents cas rencontrés. Nous vous donnerons notre point de vue sur les indications, la technique chirurgicale avec sa voie d'abord, le choix des greffes utilisées, le délai de cicatrisation, la mise en place de membrane, l'utilisation de plasma riche en plaquettes (PRP) ou de plasma riche en fibrine (PRF) et la pose immédiate ou différée des implants.

\section{TECHNIQUES CHIRURGICALES DES GREFFES OSSEUSES SINUSIENNES}

Nous avons deux possibilités de techniques chirurgicales : la voie latérale ou la voie crestale déjà décrites par Tatum.

Nous vous décrirons le cas typique d'une greffe allogénique intrasinusienne.

Dans tous les cas, un assainissement parodontal est indispensable avant l'acte chirurgical pour éliminer toutes les sources d'infection (fig. 1).

Avant l'intervention, le patient est mis sous anti-inflammatoire stéroïdien (environ 40 à $60 \mathrm{mg}$ de Solupred par jour), sous antibiotique généralement un gramme d'Augmentin deux fois par jour ou en cas d'allergie à la pénicilline, de la Pyostacine $500 \mathrm{mg}$ deux fois par jour à continuer pendant 10 jours après la chirurgie et une sédation orale généralement de l'Atarax ou du Valium.

Le patient se lave les dents à l'Hexomédine avant l'intervention. Un drapage stérile permet d'éviter une contamination externe du greffon.

L'anesthésie locale se fait à l'articaïne à $4 \%$ ou de la Xylocaïne $2 \%$ avec de l'adrénaline pour diminuer le saignement. Les infiltrations se font en vestibulaire et palatin.

L'incision est réalisée à distance de la fenêtre osseuse environ à $10 \mathrm{~mm}$ en mésial et distal. Celle-ci est d'autant plus palatine que le sinus est procident mais malgré tout pas trop palatine pour éviter une nécrose gingivale. Une incision de décharge antérieure est

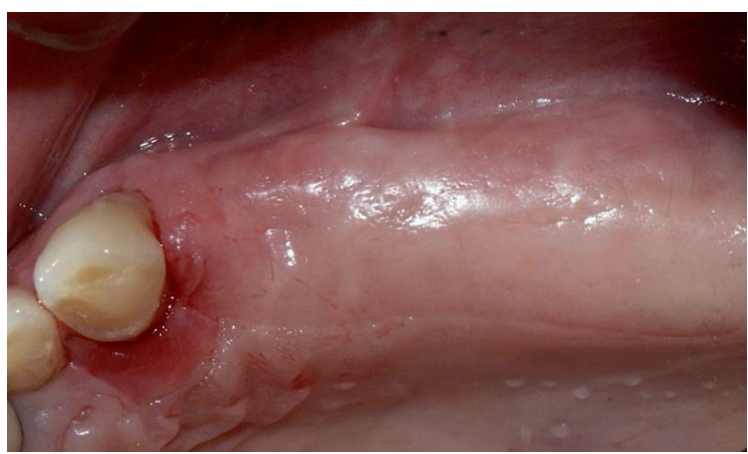

Fig. 1 :

Assainissement de l'os alvéolaire et du parodonte. 


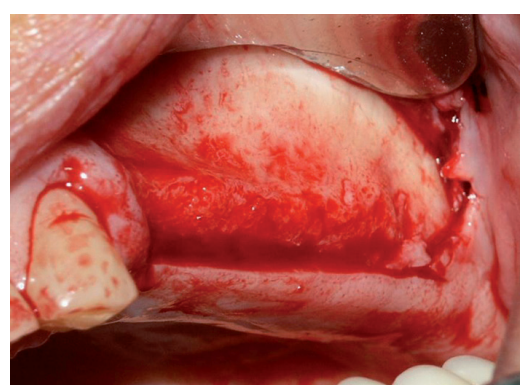

$\triangle$ Fig. 2 :

Incision à distance du volet osseux et paramarginale à distance de la dent puis décollement sous-périosté du lambeau muqueux.

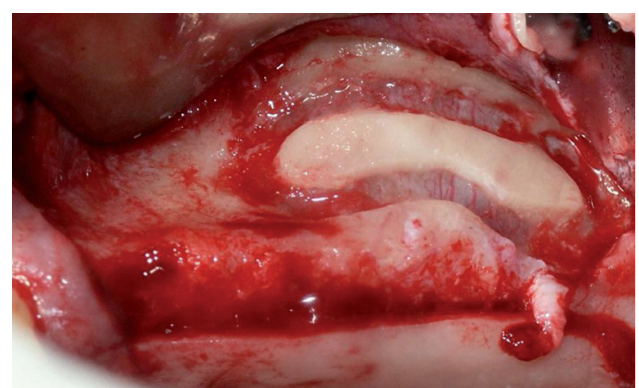

$\triangle$ Fig. 3 :

Volet osseux réalisé à la fraise boule diamantée jusqu'à apercevoir l'aspect bleuté de la membrane sinusienne qui est ensuite décollée.

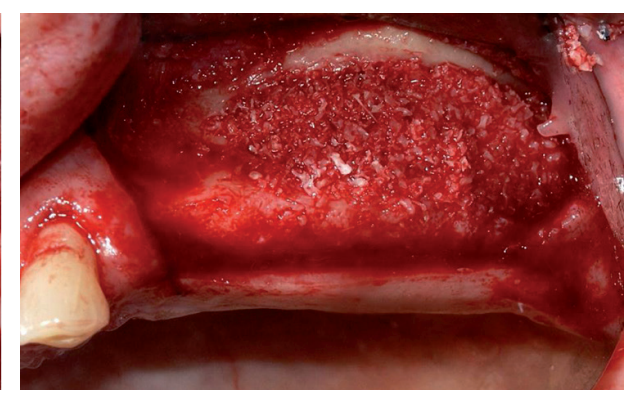

$\triangle$ Fig. 4 :

Comblement de l'espace situé sous la membrane par l’os bovin déprotéiné (Bio-oss ${ }^{\circ}$ ). généralement nécessaire, soit intrasulculaire, soit paramarginale en distal de la dent si celle-ci est dévitalisée. En tous les cas, en distal de la canine, si les autres dents sont manquantes.

Un lambeau sous-périosté est décollé soigneusement supérieurement, antérieurement et postérieurement au volet osseux (fig. 2). Ce décollement sera d'autant plus soigneux qu'une intervention précédente a été faite, soit une extraction emportant un bloc osseux, soit une ostéotomie afin d'éviter une déchirure muqueuse sinusienne.

Le volet osseux est suffisamment large pour accéder au sinus et au décollement de la membrane sinusienne mais aussi le petit possible pour conserver le maximum de cellules ostéogéniques pour la régénération osseuse. Ce volet osseux est fait à la fraise diamantée (fig. 3) ou piézotome jusqu'à voir transparaître l'aspect bleuâtre de la muqueuse afin d'éviter une déchirure de la membrane sinusienne. Lartère antrale du sinus sera repérée sur l'imagerie et cliniquement. Le volet est alors soit décollé et conservé précieusement, soit refoulé vers l'intérieur du sinus tout en décollant la membrane tout autour de la fenêtre osseuse. La membrane doit être décollée en dedans pour récliner la muqueuse vers le haut permettant le comblement du fond sinusien par des biomatériaux ou d'os autogène nécessitant dans ces cas un prélèvement. Certains auteurs comptent sur le caillot sanguin pour avoir une régénération de l'os [7]. Quand il existe une cloison sinusienne, un double volet est préférable de part et d'autre de cette cloison transversale. L'utilisation de décolleurs dont les courbures sont variables est indispensable. L'utilisation de décolleurs larges est souvent préférable pour éviter les déchirures. Le bout du décolleur sera toujours au contact de l'os.

En cas de déchirure membranaire, une membrane résorbable est utilisée. Si la déchirure est très importante, il est parfois nécessaire de remettre le comblement à quelques mois et de refermer la muqueuse gingivale.

Le comblement se fait antérieurement, médialement, postérieurement et enfin le tassement remplit la cavité qui doit correspondre à la zone à implanter. Le tassement doit être suffisant mais pas trop appuyé pour éviter les lacunes et permettre la régénération osseuse (fig. 4). Le décollement de la membrane sinusienne doit intéresser la paroi interne de la cavité du sinus pour éviter un récessus muqueux pouvant être responsable d'infection (fig. 5 et 6).

La fermeture de la fenêtre nécessite la mise en place d'une membrane résorbable ou non mais la remise en place du volet osseux suffit pour obtenir une régénération osseuse.

La pose des implants dans le même temps est possible quand généralement la stabilité primaire est assurée. Le forage est fait après le décollement de la membrane sinusienne. Sinon les implants sont posés 4 à 6 mois après la mise en place de la greffe.

La fermeture de la muqueuse gingivale est hermétique. Parfois, les implants ne sont pas enfouis quand la greffe n'est pas trop importante et que les implants ont une bonne stabilité primaire.
Fig. 5 : Décollement de la membrane sinusienne, notamment de sa paroi interne, et comblement par du Bio-oss ${ }^{\circledast}$.
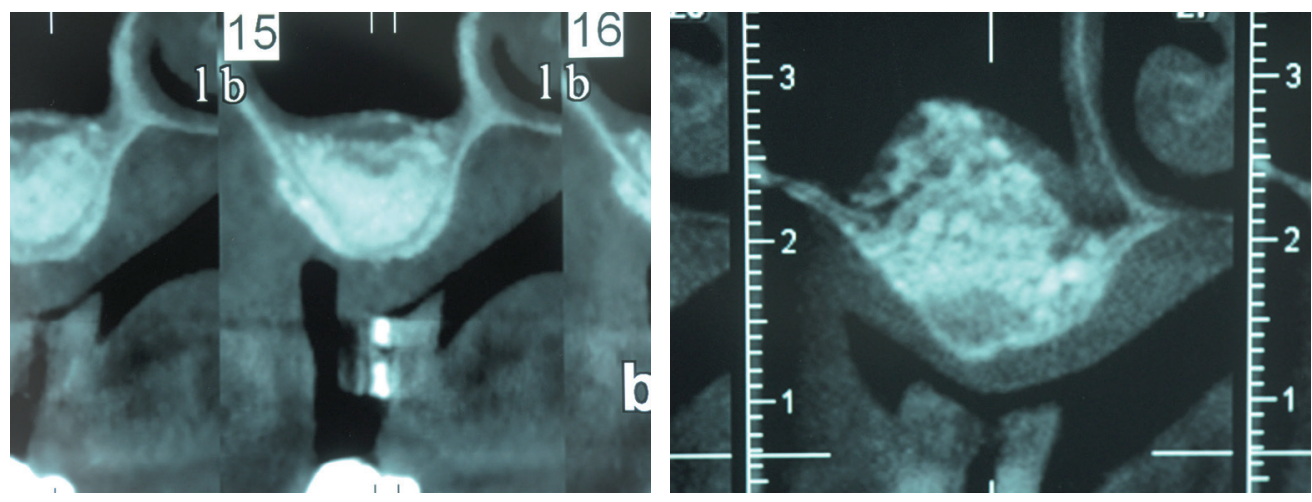

4 Fig. 6 : Décollement insuffisant de la paroi interne du sinus. 


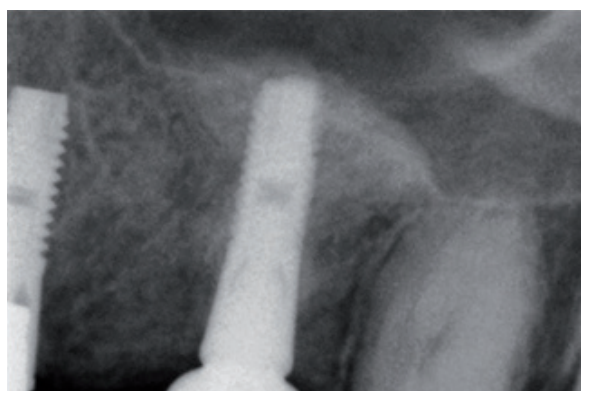

4 Fig. 7 :

Greffe de Bio-oss ${ }^{\circledast}$ par voie crestale (technique de Summers).

Les suites opératoires sont marquées par un œdème qui apparaît dans les deux jours après l'acte chirurgical. Des saignements de nez sont fréquents et le mouchage est à éviter. Les bains de bouche seront doux pendant 10 à 15 jours et le brossage des dents proche de l'incision muqueuse est à proscrire pendant ce temps. Le traitement antibiotique est prolongé pendant 10 à 15 jours. Des antalgiques sont parfois nécessaires. La prise d'anti-inflammatoires est prolongée pendant quelques jours. Lalimentation est semi-liquide pendant 10 à 15 jours en fonction de l'importance de la greffe.

Dans certains cas, la voie d'abord crestal " osseuse " est intéressante et connue sous le nom de Summers [8]. Le principe est simple, il consiste à soulever le plancher du sinus et sa membrane en regard de l'implant à poser. Si le gain nécessaire est de moins de $3 \mathrm{~mm}$ environ, il n'est pas utile d'y associer une greffe, le seul caillot sanguin suffit à la régénération osseuse. La difficulté est de ne pas perforer la membrane sinusienne. Son risque est d'autant plus faible que plusieurs implants adjacents sont posés. Il est souvent préférable d'utiliser des implants coniques. La hauteur d'os résiduel est d'au moins 6-7 $\mathrm{mm}$ avec une largeur suffisante pour réaliser la levée de la membrane avec un maximum de sécurité afin d'éviter sa perforation. Cette technique nécessite l'utilisation de fraises boules et d’ostéotomes de Summers. Des radios rétro-alvéolaires sont parfois utiles en cours de procédure pour évaluer la hauteur de fraisage notamment (fig. 7).

\section{QUEL TYPE DE GREFFE CHOISIR?}

Une greffe osseuse est nécessaire quand existe un manque de volume verticalement et parfois horizontalement du fait d'une résorption osseuse après extraction. Les greffes osseuses autologues ont longtemps été utilisées pour leurs capacités ostéoinductrices et ostéogéniques. Ces greffes sont considérées par de nombreux auteurs comme le matériel de choix, notamment quand il s'agit d'apposition osseuse. Elles ont l'inconvénient de leur prélèvement intrabuccal ou extrabuccal, crânien ou iliaque [8]. Les prélèvements intrabuccaux se font à la symphyse mentonnière ou dans la zone de l'angle mandibulaire et de sa branche montante. Le prélèvement est réalisé au mieux au piézotome afin de perdre le moins d'os possible. Les prélèvements symphysaires entraînent souvent une hypoesthésie incisive et gingivale et à un moindre degré du menton. Les suites opératoires sont marquées par un œdème et des douleurs parfois invalidantes. Pour ce type de prélèvement, une anesthésie locale est généralement suffisante. Les prélèvements exobuccaux se font sous anesthésie générale. La prise de greffe iliaque a pour inconvénient ces douleurs qui cèdent 8 à 15 jours après l'acte chirurgical.

Les allogreffes provenant de cadavre humain sont intéressantes en cas d'apposition osseuse.

Dans tous les cas d'apposition osseuse, la greffe doit être immobilisée pour assurer sa revascularisation.

Les greffes alloplastiques comme l'hydroxyapatite et le tricalcique de phosphate peuvent être utilisées pour la régénération osseuse $[10,11]$.

Actuellement, le praticien utilise volontiers des matériaux de substitution dont les résultats sont très performants. Nous les utilisons pour les comblements de sinus, notamment l'hydroxyapatite d'origine bovine, Bio-oss ${ }^{\circledR}$.

\section{UTILISATION D'UNE MEMBRANE}

Dans les cas de comblement de sinus par voie latérale, il semblerait que la mise en place d'une membrane non résorbable ou mieux résorbable permette une meilleure régénération osseuse et survie implantaire (fig. $\mathbf{8}, \mathbf{9}, \mathbf{1 0})$. Cependant, quand le volet osseux peut être

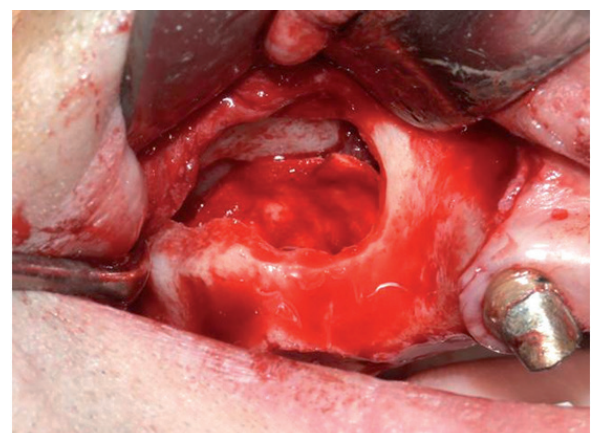

$\triangle$ Fig.8 :

Réalisation d'une greffe osseuse sinusienne par voie latérale. Ouverture du sinus et soulevé de la membrane de sinusienne.

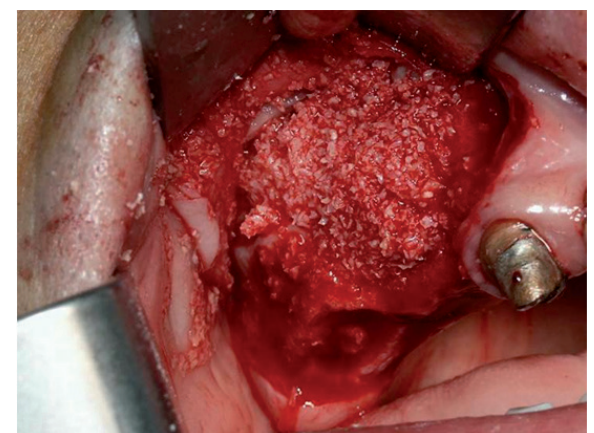

$\triangle$ Fig. 9 :

Comblement au Bio-oss ${ }^{\circledast}$ de l'espace mort situé sous la membrane.

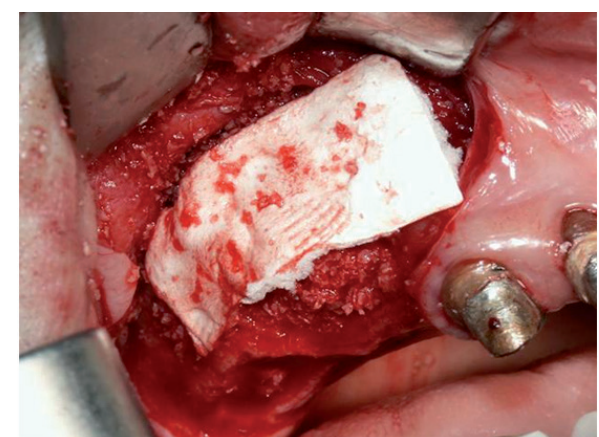

$\triangle$ Fig. 10 :

Mise en place d'une membrane résorbable (Bio-Gide ${ }^{\circledast}$ ). 
replacé, il nous paraît inutile de mettre une membrane. Les greffes osseuses autogènes ne nécessitent pas de membrane quand lopérateur utilise les corticales amincies.

\section{APPORT DE PRF}

L'utilisation de matrice de fibrine riche en plaquettes servirait de protection et d'amélioration de la cicatrisation. Elle est obtenue par la centrifugation du sang. Son rôle n'est pas encore retenu et il n'existe pas de consensus.

Nous vous présentons quelques cas de greffes osseuses sinusiennes.

I $1^{\text {er }}$ cas. Patient présentant une édentation dans le secteur I et II, le secteur incisivo-canin est conservé (fig. 11).

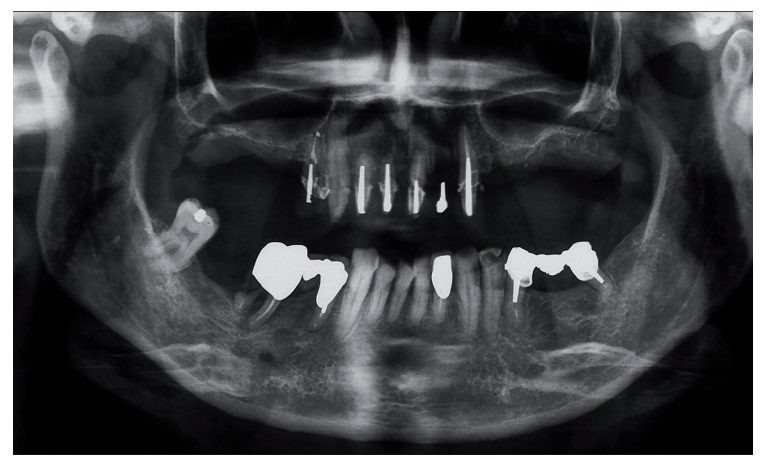

$\triangle$ Fig. 11 :

Panoramique dentaire montrant l'édentation et l'absence de hauteur osseuse.
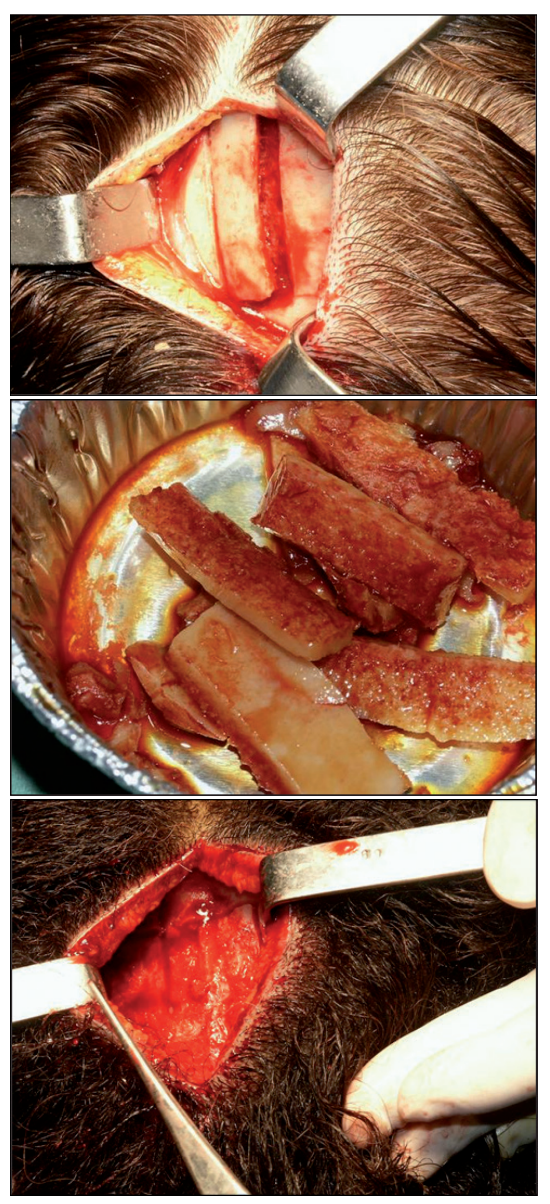

4 Fig. 12,

13 et 14 :

Prélèvement

de la corticale

externe de l'os pariétal droit.

La perte de

substance est

comblée par de

la résine auto-

polymérisante.
Une greffe osseuse autogène est choisie devant l'absence quasi totale de hauteur osseuse sinusienne. Le prélèvement est réalisé en pariétal crânien droit (fig. $12,13,14)$.

Un volet osseux latéral est confectionné à la fraise diamantée et déposé. La membrane est alors soulevée jusquà la paroi interne du sinus pour avoir le maximum de contact osseux avec la paroi du sinus. (fig. $15,16)$.

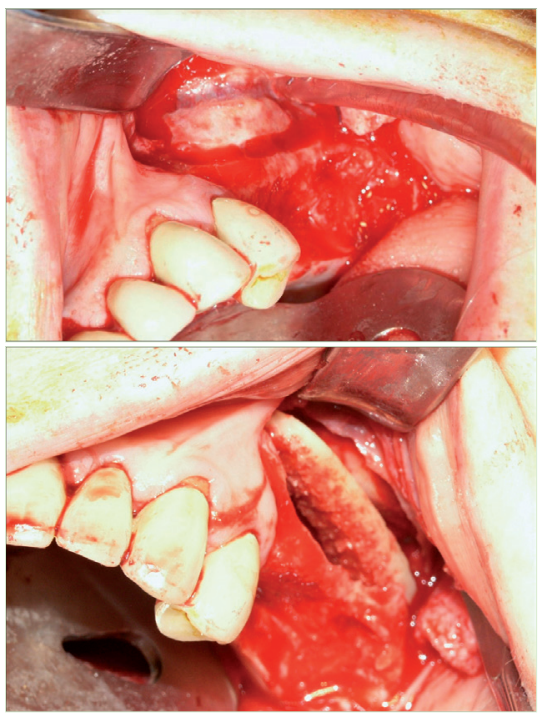

4 Fig. 15 et 16 : Ouverture du sinus à la fraise diamantée et comblement d'os broyé en reconstruisant le plancher du sinus avec de la corticale.

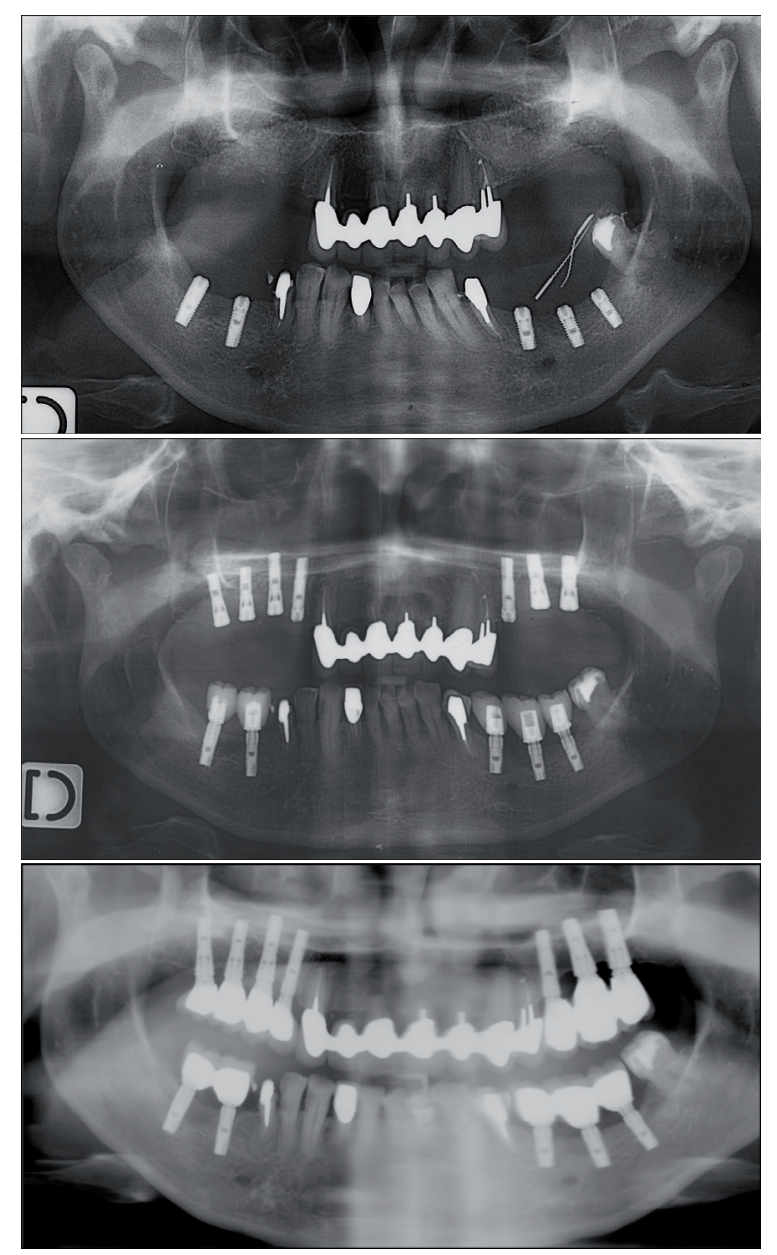

$\triangle$ Fig. 17, 18 et 19 :

Panoramiques dentaires montrant la prise de greffe, la pose des implants et ensuite les prothèses. 


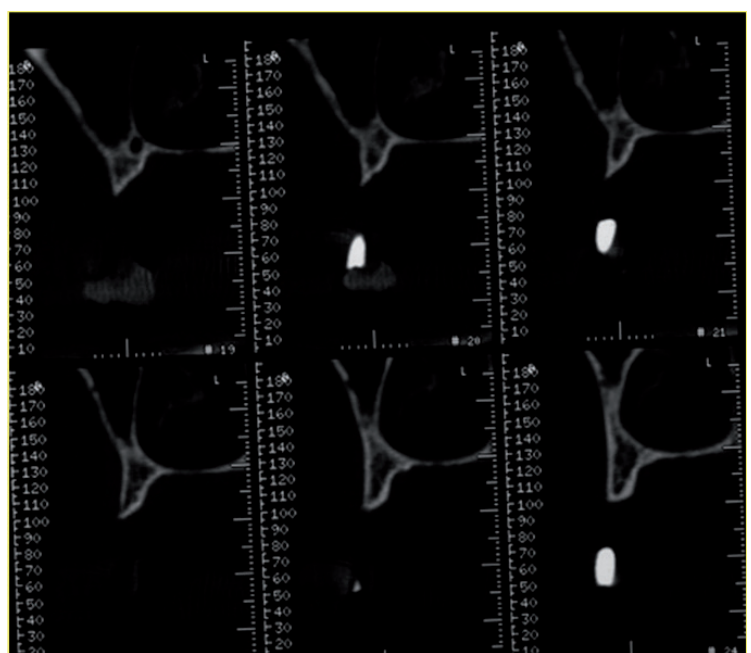

$\triangle$ Fig. 20 :

Radios montrant la perte osseuse verticale et transversale maxillaire.

Des implants à surface rugueuse sont posés six mois après la greffe puis les prothèses cinq mois après ces implants (fig. 17, 18, 19).

Les greffes sinusiennes " osseuses » ont transformé l'implantologie au niveau du maxillaire. L'utilisation de biomatériaux est de plus en plus fréquente avec souvent la pose d'une membrane résorbable. En revanche, les greffes osseuses autogènes voire les allogreffes sont préférées en cas d'apposition et d'absence quasi totale de hauteur osseuse résiduelle.

1 $2^{\mathrm{e}}$ cas. Patient présentant une perte osseuse verticale et transversale du maxillaire (fig. 20). Une greffe osseuse d'apposition est associée à une greffe d'apposition permettant d'augmenter le sens transversal maxillaire (fig. 21).

$13^{\mathrm{e}}$ cas. Patiente présentant un kyste apico-dentaire de la 26 et une perte osseuse verticale (fig. 22). Il s'agit d'un cas typique d'extraction de la 26 et assainissement alvéolaire (fig. 23 et 24). Deux à trois mois après cet acte, une greffe osseuse de Bio-oss ${ }^{\circledast}$ est faite par voie latérale avec pose immédiate des implants non enfouis. L'os résiduel permet une stabilité primaire. (fig. 25 et 26).

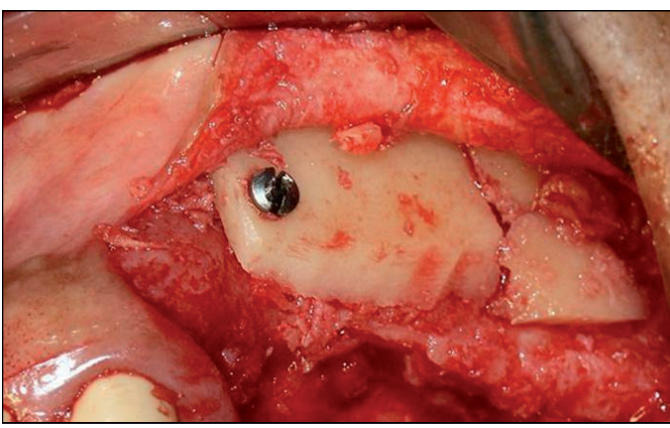

$\triangleleft$ Fig. 21

Mise en place d'un greffon d'apposition pour augmenter le sens transversal maxillaire. L'immobilisation du greffon est primordiale pour la prise de greffe.

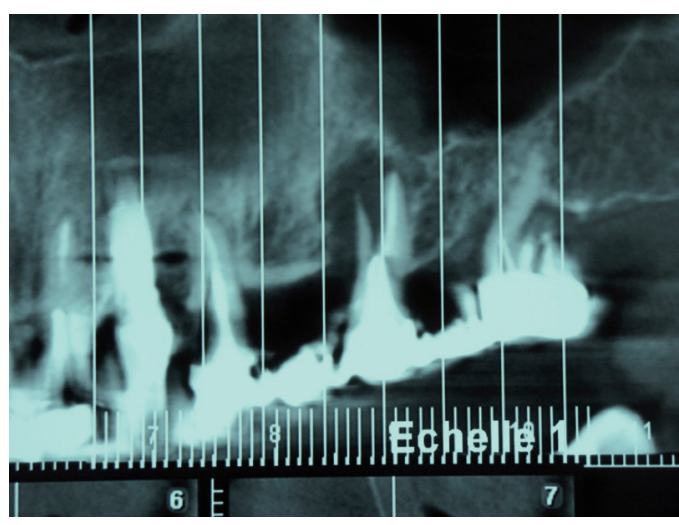

$\triangle$ Fig. 22 :

Granulome apicodentaire de la 26 et perte osseuse verticale.

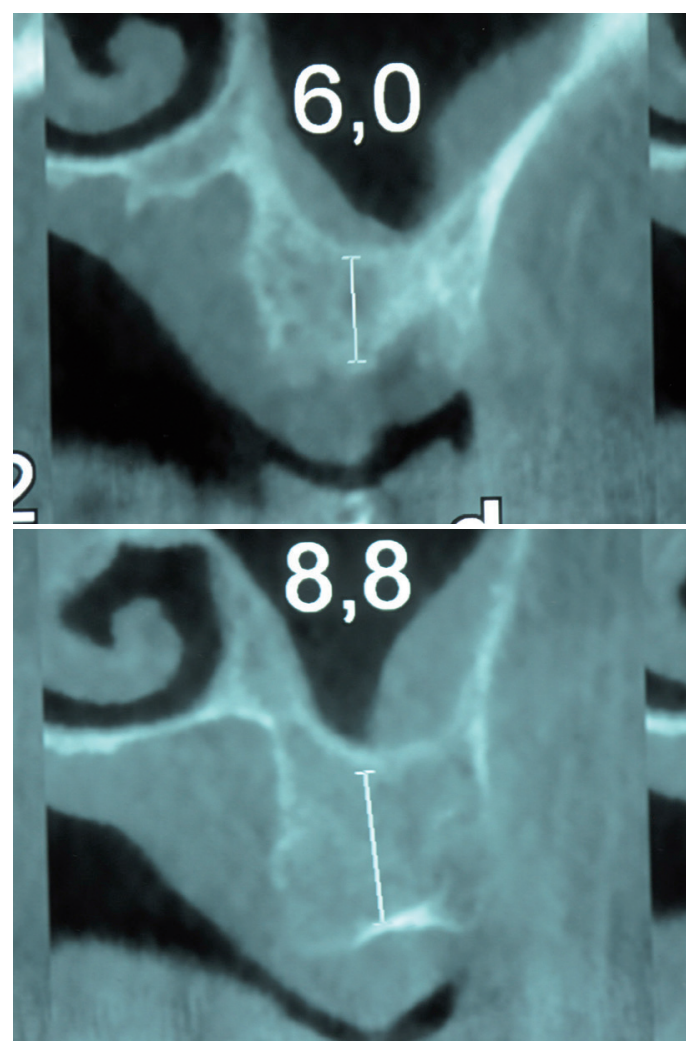

4 Fig. 23 et 24 :

Assainissement avec extraction. Les coupes verticales de cone-beam montrent l'absence de volume osseux.
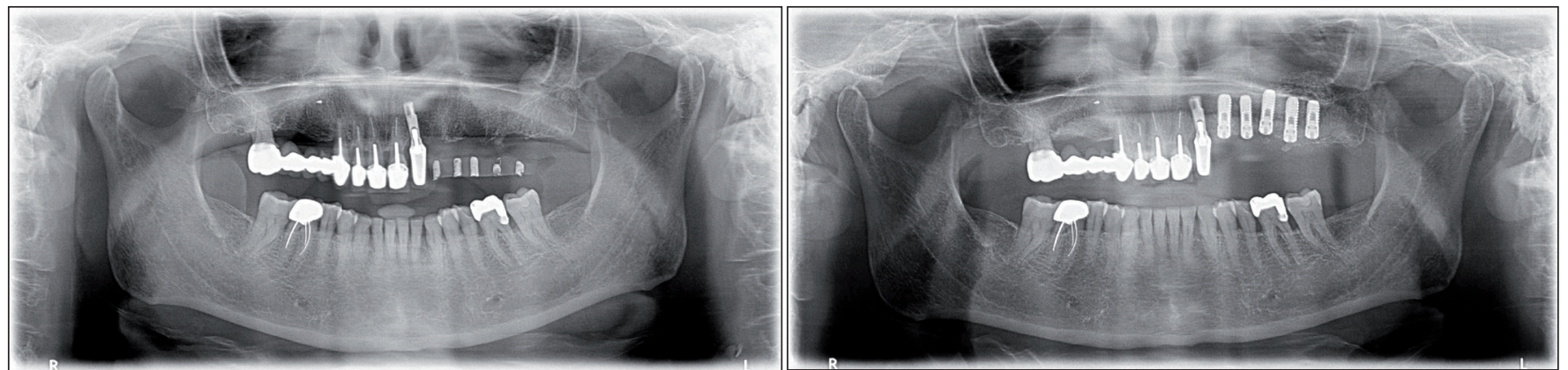

Fig. 25 :

Panoramique dentaire après extraction de la 26 et assainissement de l’os alvéolaire puis greffe de Bio-oss ${ }^{\circledR}$ et pose d'implants. 


\section{Bibliographie}

[1] Terracol J, Ardouin P. Anatomie des fosses nasales et des cavités annexes. Paris : Maloine, 1965.

[2] Rosano G, Taschieri S, Gaudy JF, Lesmes D, Del Fabbro M. Maxillary sinus septa: a cadaveric study. J Oral Maxillofac Surg 2010;68:1360-1364.

[3] Geha N, Carpentier P. Arterial loops in the maxillary sinus. J Parodontol Implantol Orale 25:127.

[4] Carpentier P, FelizardoR, Cledes G. Anatomie des techniques anesthésiques intra-orales. Réalités Cliniques 2006;17:159-176.

[5] Sharan A, Madjar D. Maxillary sinus pneumatization following extractions: a radiographic study. Int $J$ Oral Maxillofac Implants 2008;23:48-56.
[6] Tatum OH Jr. Maxillary and sinus implant reconstructions. Dent Clin North Am 1986;30:207-229.

[7] Nedir R, Bischof M, Vasquez L, Nurdin N, Szmukler-Moncler S, Bernard JP. Osteotome sinus floor elevation technique without grafting material: 3-year results of a prospective pilot study. Clin Oral Implants Res 2009;20:701-707.

[8] Summers RB. A new concept in maxillary implant surgery: the osteotome technique. Compend Contin Educ Dent 1994a;2:152-160.

[9] Tessier P. Autogenous bone grafts taken from the calvarium for facial and cranial applications. Clin in Plast Surg 1982;9:531-538.
[10] Antoun H, Sitbon J, Martinez H, Missika P. A prospective randomized study comparing two techniques of bone augmentation: onlay graft alone or associated with a membrane. Clinical Oral Implants Research 2001;12:2-639.

[11] Wallace SS, et al. Sinus augmentation utilizing anorganic bovine bone (Bio-oss) with resorbable and nonresorbable membranes placed over the lateral window: histomorphometric and clinical analyses. Int Periodontics Restorative Dent 2005;25:551-559.

\section{Analyse}

\section{Crétôt $\mathrm{M}$.}

L'arcade dentaire humaine. Morphologie.

Huitième édition remaniée. Éditions CdP, Rueil-Malmaison, 2013.

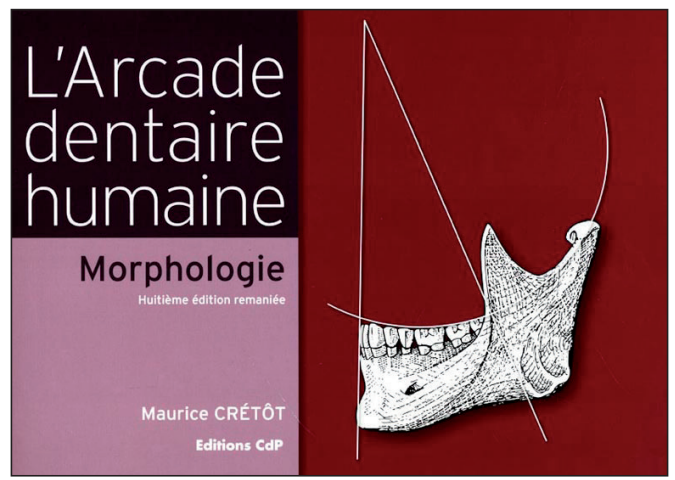

Ma génération a été abreuvée à la mamelle de Tallec et de Marguerite et de leurs dessins de morphologie dentaire. La génération actuelle se régale (!), dans ses premières années d'étude, des dessins de Maurice Crétôt. Les premières éditions se basaient sur des moulages en plâtre de nombreuses arcades dentaires «normales» (mot difficile d'emploi aujourd'hui...) et de radiographies. Cette huitième édition ajoute des renseignements supplémentaires apportés par l'analyse de 275 téléradiographies d'adultes de 18 à 60 ans. Ces nouvelles références ont permis de modifier l'orientation de certains éléments sur quelques planches globales.

Outre la morphologie «normée» des dents, cet ouvrage aborde l'engrènement des arcades avec les points d'impact respectifs, les courbes d'occlusion, les plans sagittaux, les rapports des arcades dentaires avec la face, les trajectoires de mastication, les rapports de certaines dents avec les parties molles et des notions sur l'embryologie et l'évolution des dents avec le temps. On le voit, un ouvrage complet qui m'a replongé dans mes jeunes années d'étudiant en chirurgie dentaire où l'on apprenait par coeur les cotes des dents (que certains d'ailleurs planquaient sous leur montre..., j'en ai vu...) et où nous nous efforcions de reproduire ces dessins lors de l'examen de fin d'année avec des résultats divers. Mais cette connaissance s'est révélée être indispensable par la suite pour l'exercice de notre Art (mot que je préfère toujours à celui de médecine dentaire) qui, outre des connaissances médicales nécessaires, reste un acte manuel dont l'apprentissage nécessite de se «dégourdir» les doigts à l'aide, entre autre, de dessins. C'est un point de départ indispensable que Maurice Crétôt permet de faire à des générations d'étudiants. Vivement la neuvième édition, gage de la vitalité de cette discipline.

Marc BERT 